\title{
Approach to detecting latent defects of the printed circuit boards thin-filmed coatings
}

\author{
Alexey Balastov ${ }^{1, *}$, Boris Kovrigin ${ }^{1}$, and Valery Lavrinovich ${ }^{2}$ \\ ${ }^{1}$ National Research Tomsk State University, 634050 Tomsk, Russia \\ ${ }^{2}$ All-Russian Scientific Research Institute of Technical Physics, 456770 Snezhinsk, Russia
}

\begin{abstract}
One of the main reasons for the space vehicles (SV) power supply systems failure is a secondary arc. In the statistics of fatal failures that can lead to the SV loss, the failure rate of the power supply systems is $9 \%$, but despite this, there are no standard methods for diagnosing the onboard equipment for the arc stability, due to the relative novelty of the problem. The problem we encountered is associated with the need to switch power supply systems to the voltage levels that are much higher than the arc voltage in the space vacuum. From the point of view of the defects downing that can lead to arcing, the most vulnerable are the insulation coatings. Therefore, a significant part of the problem solution is concentrated in the development of the specific methods for these coatings diagnostics. In space technology, such diagnostics significance is increased due to the inability to provide necessary repairs or services in the outer space.
\end{abstract}

\section{Introduction}

The transition to the new operating voltage standard in satellite electronics has led to the problem of arcing between the printed circuit board elements. In order to keep the mass of the satellite as small as possible, the space inside the circuit housing is not filled with an insulator or is not sealed. It contributes to the emergence of electric discharges, which could potentially extend to the satellite on-board equipment. The ignition of the electric arc in the onboard satellite system always leads to failure. In most cases, it leads to the satellite termination. This research main aim is to improve the quality of the satellites on-board electronic equipment. The final result of the research is a proven and patented installation and method for determining the printed circuit boards thin-filmed coatings defects for the satellites on-board electronic equipment. The obtained results will be used to develop documentation and prototypes testing.

\section{How it works?}

Insulation coatings are the most vulnerable to arcing. Therefore, a significant part of the problem's solution is concentrated in the development of a new insulation coatings inspection

\footnotetext{
* Corresponding author: lexiusb@gmail.com
} 
method. The corona discharge phenomenon is known for a long time. There are many practical and theoretical materials explaining the nature and properties of the corona discharge [1]. We have proposed the possibility of finding latent defects in the printed circuit boards (PCB) coatings, based on the corona discharge properties, using a reverse corona, that can be registered visually or via ampermeter. Each defect in the insulation coating is a good conductive channel [2]. Our installation can register this conductivity due to the corona discharge properties.

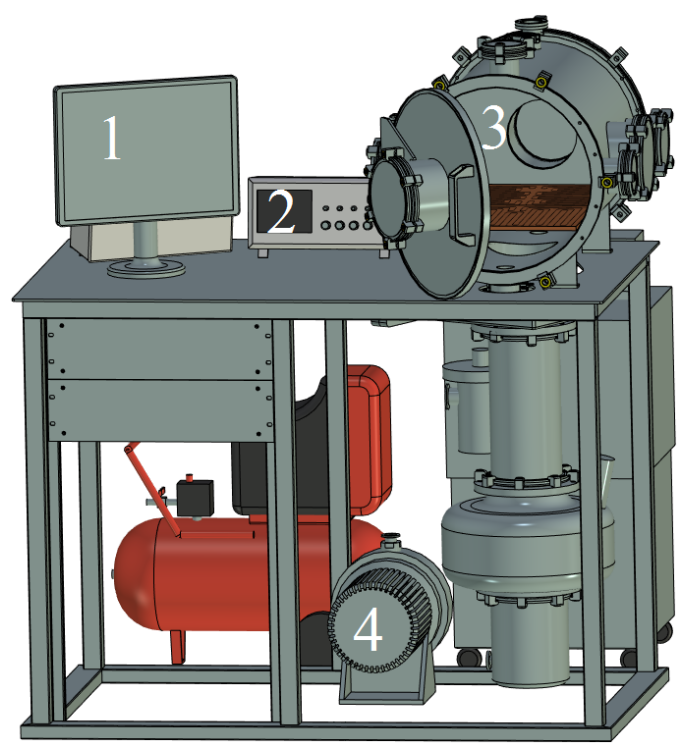

Fig. 1. T-Flex CAD model of the developed installation: 1 - Computer; 2 - Oscilloscope; 3 - Vacuum chamber; 4 - Vacuum pump.

PCB (4) is placed between point-plane system with different potentials ( 3 and 5). Thermal electron emission gives sufficient number of electrons to generate a little electrical current. If insulation coating has a defect, we can register it via the ampermeter (6).

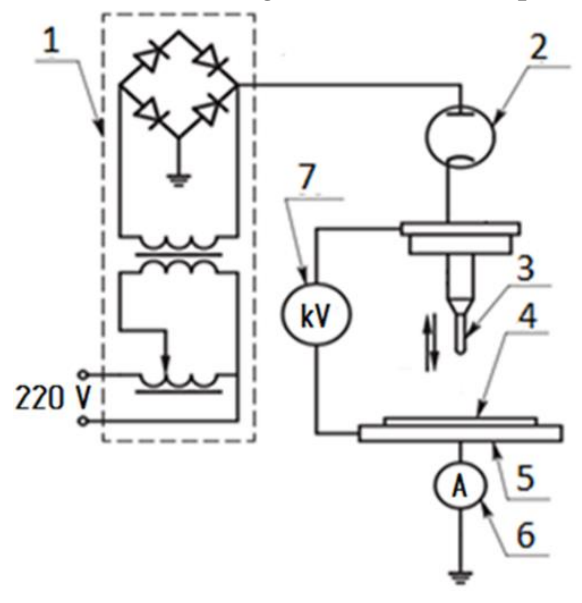

Fig. 2. Diagnostic electric scheme.

There are given types of defects that can be detected by corona discharge testing: 

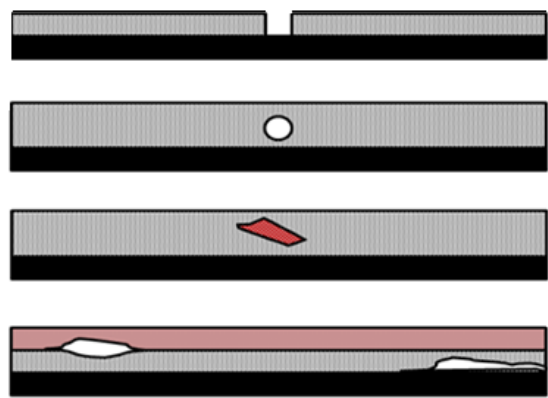

Delamination

Fig. 3. Type of defects.

\section{Glowing effect}

High voltage corona discharge can glow. When it happens, this glowing area covers the defected zone and the way from the point to the zone. This simple property provides good $\mathrm{PCB}$ coating defects visualization.

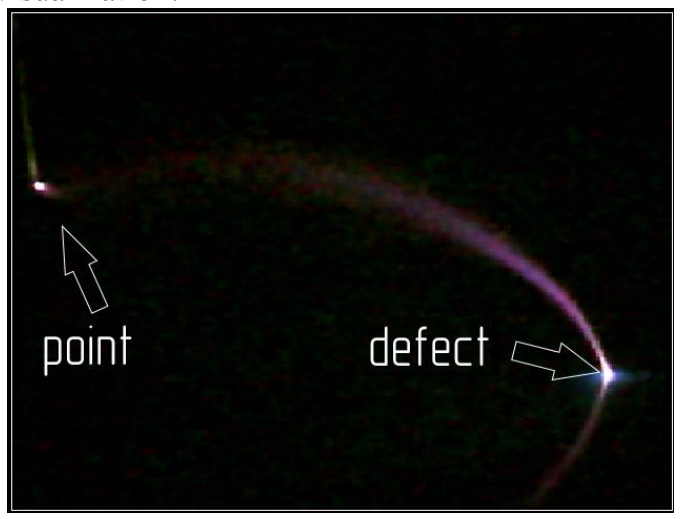

Fig. 4. Glowing area for 1 defect.

The glow effect will work even if the printed circuit board has more than one defect. This feature allows us to re-evaluate the potential of this testing method. Using the corona discharge it is possible to determine not only the defect presence, but also to localize it with high accuracy [3].
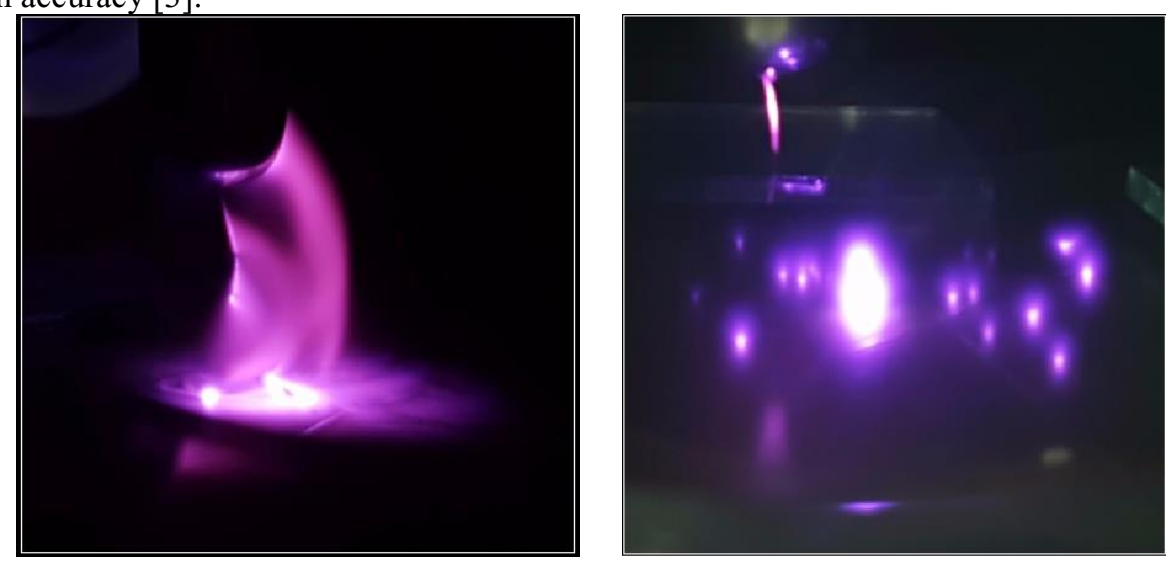

Fig. 5. Glowing area for 3 defects (left) and for 14 defects (right). 


\section{The research preliminary results}

There can 't be used tests that can decrease PCB service life because of the satellite electronics high complexity and cost. Limiting the choice of diagnostic methods and providing testing only via the nondestructive one, significantly reduces the range of possible diagnostic methods application. An additional condition is the method's ability to determine various types of insulation defects and to provide testing object entire surface diagnostics, including when the method is applied to the printed circuit boards consisting of a large number of elements and complex topology.

The purpose of the first experiment was the detection of a specially made defect in the test sample insulation coating.

There were obtained the following results:

- $\quad$ The defect registration occurs before reaching the breakdown voltage;

- There is enough $1 \mathrm{kV}$ voltage in order to detect a defect;

- There is recorded current from 10 up to $50 \mu \mathrm{A}$ if the insulating coating has got a defect;

- There is a clear possibility to detect multi-defects and to limit the breakdown current.

\section{Conclusion}

The use of a controlled corona discharge as a diagnostic tool, in our opinion, will make it possible to: exclude a contact with the dielectric coating for detecting latent defects; reduce testing voltage to $1 \mathrm{kV}$ and lower; reduce testing current in the circuit up to microamps. It is necessary to note that the defect determination always occurs before the breakdown. Therefore, the corona discharge testing can be referred to the nondestructive testing methods.

There are some plans for the further studies:

1. Automate the process of detection and lower the test voltage;

2. Determine the experiment optimal parameters;

3. Upgrade the installation;

4. Conduct further experiments.

\section{References}

1. R. Islam, P. D. Pedrov, Shuzheg Xie, K. R. Englund, Trans. Plasma Sci. 43, 3695 (2015)

2. J.J. Lowke, R. Morrow, Pure \& Appl. Chem. 66, 1287 (1994)

3. V.A. Lavrinovich, Bulletin of the Tomsk Polytechnic University. Geo Assets Engineering 327, 58 (2016) 